\title{
Stochastic Vehicle Routing Problem: A Literature Survey
}

\author{
Eshetie Berhan*, Birhanu Beshah ${ }^{\dagger}$ and Daniel Kitaw* \\ School of Mechanical and Industrial Engineering \\ Addis Ababa Institute of Technology, Addis Ababa University \\ Addis Ababa, Ethiopia \\ *eshetie_ethio@yahoo.com \\ †bireabesh@yahoo.com \\ †danielkitaw@yahoo.com \\ Ajith Abraham \\ Machine Intelligence Research Labs (MIR Labs) \\ Scientific Network for Innovation and Research Excellence \\ Washington, USA \\ abraham.ajith@gmail.com
}

Published

\begin{abstract}
The purpose of this paper is to develop structural classification of Stochastic Vehicle Routing Problem (SVRP) by different domains and attributes. This research used a systematic review and meta-analysis on SVRP literatures. This includes browsing relevant researches and publications, screening related articles, identifying domains, attributes and categorising the articles based on the identified domains and attributes. The findings of the study show clear differences on the number of studies under each domain and attribute. Most studied attributes are stochastic customer demand, capacitated vehicle, synthesis data and objective function with cost minimization. Whereas the least studied are maximisation objective function, stochastic service time, and an applied model using stochastic with recurs. The research helps to summarise and map a comprehensive survey on SVRP literatures so that various contributions in the field are organised in a manner that provide a clear view for the readers and identify future research directions. This paper is the first of its kind in the field of SVRP that develop a classification scheme for articles published since 1993 to enhances the development of this newly emerging discipline.
\end{abstract}

Keywords: Vehicle routing problem; stochastic VRP; classification, attributes; domain.

\section{Introduction}

The task of designing delivery or pickup routes to service customers in the field of transportation and supply chain is known in the literature as a Vehicle Routing Problem (VRP) (Christopher, 2010). Dantzig and Ramser (1959) proposed VRP for the first time under the title "Truck dispatching problem" with the objective to design optimum routing of a fleet of gasoline delivery trucks between a bulk terminal and a large number of service stations. VRP is a generic name given to a whole class of problems in which a set of routes for a fleet of vehicles based at one or several depots must be determined for a number of geographically dispersed cities or customers (Laporte et al., 1989, 2002; Calvete et al., 2004; Baldacci et al., 2010). It is defined by a depot, a set of geographically dispersed customers with demands, and a set of vehicles with capacity. In addition, customers must be visited exactly once and the total customer demand of a route must not exceed the vehicle capacity (Clarke and Wright, 1964; Laporte et al., 2002; Novoa et al., 2006). Its objective is to find a collection of $K$ simple circuits each corresponding to a vehicle route with minimum cost, distance or time.

To achieve this objective, extensive research has been conducted on VRP to design and develop different models and optimisation methods. VRP models are differed by inclusion or exclusion of different side constraints. The commonly used side constraints are restrictions on capacity of vehicle, total time, windows time, precedence relations between pairs of cities, the number of depots (Laporte, 1992; Madsen et al., 1995; Paolo and Daniele, 2002; Chenghua and Xiaofeng, 2011) and others. Basically,

\footnotetext{
${ }^{*}$ Corresponding author.
} 
VRP can be classified as classical/deterministic and stochastic. The former assumes static or deterministic parametric value in the VRP model and the latter however, considers stochastic parameters. The consideration of stochastic information on VRP models is differed from the classical VRP on approach the problem is formulated and the solution is implemented.

Due to the number of variables and side constraints considered, research in the areas of Stochastic Vehicle Routing Problem (SVRP) is growing both intensively and extensively at a rapid peace. According to the survey made by Eksioglu et al. (2009), 1494 bibliographical entities were reviewed for the purpose to survey VRP taxonomy and found that VRP literatures are growing almost exponentialy with $6.09 \%$ annual growth rate. Further, they pointed out that due to enhanced data collection, tracking capabilities, and computational availabilities, trends in the VRP literature have shifted from static to more dynamic and fuzzy cases that incorporate real time data and stochastic parameter in it. Similar surveys were previously conducted on different variants of VRP. Among them, dynamic VRP with perspective of quality and evolution (Pillac et al., 2011), VRP on particular interest of pickup and delivery problems (Parragh et al., 2008), Capacitated VRP (CVRP) VRP with Time Window (VRPTW) (Kumar and Panneerselvam, 2012), classification of VRP based on network classification and number of vehicles (Bodin, 1975) and classification scheme of VRP based on computer data structure (Desrochers et al., 1990). As it can be seen from the literatures, so far, there has not been extensive research particularly, that addresses the classification of SVRP. Due to this reason, as compared to other variants of VRP, research in SVRP is rather scattered and not organised so far in a systematic manner. This paper, therefore, tries to conduct a comprehensive survey using a systematic review and meta-analysis approach on the SVRP literatures to map the various contributions and identify patterns in a manner that provide a clear view to the readers and identify future research directions.

The remainder of this article is organised as follows: Sec. 2 gives a brief literature on SVRP, Sec. 3, states the research methodology, Sec. 4, presents the result and discussion and at last, Sec. 5, gives the conclusions and future research directions.

\section{Literature Review}

\subsection{Vehicle routing problem}

VRP is represented in a graph theory. The general model of VRP is defined as: Let $G=(v, A)$ be a asymmetric graph where $V=\{0,1, \ldots, n)$ is a set of vertices representing cities with depot located at vertex 0 , and $\mathrm{A}$ is the set of arcs. With every $\operatorname{arc}(i, j) ; i=j$ is associated a nonnegative distance matrix $C=\left(C_{i j}\right)$. In some context, $C_{i j}$ can be interpreted as a travel cost or as a travel time (Laporte, 1992; Cordeau et al., 2001; Golden et al., 2008; Wouter, 2008; Li et al., 2010). When $C$ is symmetrical, it is often convenient to replace $A$ by a set $E$ of undirected edges, the graph being called symmetric or undirected graph.

The general or classical VRP consists of designing a set of at most $K$ delivery or collection routes such that each route starts and ends at the depot, each customer is visited exactly once by exactly one vehicle, the total demand of each route does not exceed the vehicle capacity and the total routing cost is minimised. With all theses assumptions, according to Stewart and Golden (1983), a compact formulation of VRP can be written as:

$$
\text { Minimise }=\sum_{k=1}^{n} c_{i j} x_{i j}^{k}
$$

Subject to

$$
\sum_{i, j}^{n} q_{i} x_{i j}^{k} \leq Q, k=1,2, \ldots n
$$

where:

$c_{i j}=$ The cost/distance of traveling from $i$ to $j$

$x_{i j}^{k}=1$ if vehicle $k$ travels from $i$ to $j ; 0$ otherwise

$m=$ The number of vehicles available

$S_{m}=$ The set of all feasible solutions in the $m$-traveling salesman problem ( $m$-TSP)

$q_{i}=$ The amount demanded at location $i$

$Q=$ The vehicle capacity.

In many practical cases, the cost or the distance matrix satisfies the triangular inequality such that $c_{i k}+c_{k j} \geq c_{i j}$; $\forall_{i, j, k} \in V$ (Paolo and Daniele, 2002).

\subsection{Variants of VRP}

The inclusion of real life scenarios on VRP models resulted in many variants of VRP that evolved from the general VRP model. For instance, the most commonly studied variant is VRPTW (Madsen et al., 1995; Cordeau et al., 2001, 2002, 2007; Kenyon and Morton, 2003; Kallehauge, 2008). It is the same problem as VRP but with additional restriction on time called window time which associated a defined time interval wherein the customer has to be supplied (Balakrishnan, 1993; Taillard et al., 1997; Calvete et al., 2004; Stefan and Natalia, 2009; Xiangyong et al., 2010).

Consideration of time interval at the depot is also called the scheduling horizon in VRP. This consideration is similar to the case CVRP with Pick-up and Deliveries 
and time Windows (CVRPPDTW). Multiple Depot VRP (MDVRP) (Blanton and Wainwright, 1993; Mingozzi, 2003), Multiple Depot VRP with Time Windows (MDVRPTW), Split Delivery VRP with Time Windows (SDVRPTW) and Periodic VRP with Time Windows (PVRPTW) (Dror and Trudeau, 1989, 1990; Dror et al., 1994) are also other variants in the time restriction.

In addition to the above variants, more additional side constraints are also added in the problem structures that lead to have more variants of VRP. Some of them are multiple depots (Bertsimas and Van Ryzin, 1990), a paired-vehicle recurs strategy (Ak and Erera, 2007), route duration limit (Christopher, 2010; Erera et al., 2010), single vehicle and limited capacity and priority on customers' visit (Bertsimas, 1991, 1992; Smith et al., 2010). Another strategy to account for the demand uncertainties is to develop a waiting strategy for vehicles (Ghiani et al., 2008) and uncertainty demand with adverse weather conditions (Sumalee et al., 2011).

However, the classical VRP model usually does not capture an important aspect of real life transportation and distribution-logistic problems. This is due to the fact that several of the problem parameters such as demand, time, distance, and others are stochastic by their nature, but they are often oversimplified and treated as deterministic (Ak and Erera, 2007).

\subsection{Stochastic VRP}

SVRP is also a generic name given to VRP that considers one or more parameters as stochastic or unknown during the planning horizon. The SVRP refers to a family of problems, that combine the characteristics of stochastic and integer programs, and are often regarded as computationally intractable (Gendreau et al., 1996). The random parameters may be the presence of customers, the nature of customer demand at a given location, the time such as service time, or travel time or windows time (Reimann, 2005).

As shown in Table 1, most common variants of SVRP are VRP with stochastic customer demands (Dror and Trudeau, 1989; Laporte et al., 1989; Yang et al., 2000; Novoa et al., 2006; Smith et al., 2010), stochastic customers (Bertsimas, 1991), stochastic customer and demand (Dror, 1993; Dror et al., 1993; Gendreau et al., 1995; Dror and Trudeau, 1986) and stochastic service time (Laporte, 1992). SVRP also addresses other areas such as unknown knowledge of the road conditions, passengers shift and working hours, heterogenous vehicles, split customer demand, number and size of vehicle, and others.

Table 1 shows a brief summary of previous SVRP studies that considered stochastic parameters and other side constraints. It presents an overall view of different

Table 1. Brief summary of literatures related to SVRP.

\begin{tabular}{|c|c|}
\hline General characterstics & Special factors considered \\
\hline $\begin{array}{l}\text { Stochastic Customer } \\
\text { Demand }\end{array}$ & $\begin{array}{l}\text { - Using the Cross-Entropy Method (Yang et al., 2000; Chepuri and Tito, 2005) } \\
\text { - With restocking (Yangoural et al., 2000) } \\
\text { - Dynamic VRP With multiple vehicles priority (Smith et al., 2010) } \\
\text { - With Euclidian service region (Bertsimas, 1992; Bertsimas and Van Ryzin, 1990, 1993) } \\
\text { - With different route updating strategies (Bertsimas, 1991) } \\
\text { - With paired-vehicle recurs strategy (Ak and Erera, 2007) } \\
\text { - Using ant-based simulation (Rossetti et al., 2009) } \\
\text { - With dynamic or re-optimisation perspective (Secomandi and Margot, 2007; Novoa and Storer, 2009) } \\
\text { - (Dror, 1991; Erera and Daganzo, 2003; Ismail and Irhamah, 2008; Milić and Jovanović, 2011) } \\
\text { - With duration constraints (Erera et al., 2010) } \\
\text { - WRP with stochastic demands (Novoa et al., 2006) } \\
\text { - Using advanced Particle Swarm Optimisation (PSO) (Moghaddam et al., 2012; Yong and Hai-Ying, } \\
\text { - With uncertainty and omitted customers (Waters, 1989) } \\
\text { - With a known probability distribution and customer revisited (Bastian and Rinnooy, 1992) } \\
\text { - And Restricted Failures (Dror et al., 1993) }\end{array}$ \\
\hline $\begin{array}{l}\text { Customer and } \\
\text { Customer Demand }\end{array}$ & $\begin{array}{l}\text { - With uncertain customer presence (Guo, 2004) } \\
\text { - With known probability distribution (Gendreau et al., 1995) } \\
\text { - Uncertain demand and adverse weather condition (Sumalee et al., 2011) }\end{array}$ \\
\hline Service Time & $\begin{array}{l}\text { - With random travel and service time (Kenyon and Morton, 2003) } \\
\text { - With stochastic travel and service times (Campbell and Gendreau, 2011) } \\
\text { - Under stochastic service times (Cortés et al., 2007) }\end{array}$ \\
\hline
\end{tabular}




\section{E. Berhan et al.}

SVRP articles by major categories as general characteristics and special factors considered under each category.

Despite the progress of SVRP research so far, generic VRP review has been done by Bodin (1975), Desrochers et al. (1990), Gendreau et al. (1996), Ismail and Irhamah (2008), Parragh et al. (2008), Eksioglu et al. (2009), Pillac et al. (2011) and Kumar and Panneerselvam (2012). But have limitation of not showing clearly structure and classification on SVRP literatures. Hence, structural analysis of these research work will help to further enhance the development of this newly emerging discipline.

\section{Research Methodology}

In order to conduct the meta-analysis, this research used a systematic literature review on SVRP. After browsing relevant research and publications a systematic review was used to screen related articles, and identifying domains and attributes. Finally based on the meta-analysis, articles that are related to SVRP were categorised based on the domains and attributes so as to identify pattern and/or distribution.

\subsection{Browsing literature}

From a wide set of academic studies, Google and Google scholars search engines, Science Direct, Springer, Jstor, Emerald and DBLP database, and other free or subscribed journal were used as a source to search VRP literatures. More than 3000 bibliographical entities, which are written in English language were searched and collected using the term "Vehicle Routing", "Vehicle Routing Problem" or "Stochastic Vehicle Routing Problem" as a search phrase(s). These include journal articles, book chapters, technical reports, and articles from various conference proceedings. But for this research, the term article is used and it means any one of them. All of them were kept for initial review.

\subsection{Screening related literatures}

After the initial scan using a systematic review, 49 articles that were published since 1963 which are related to
SVRP are identified. The number of articles and year of publications for all the 49 articles are summarised and presented in Table 2.

\subsection{Identifying domains and attributes}

Using extensive survey, the systematic review resulted in eight different domains and twenty nine attributes. The identified attributes and domains are shown in Table 3. The domains include: Stochastic scenario, solution approach, data characteristics, type of graph they represent, number of vehicle, vehicle capacity, objective function, and model applied. They are introduced by considering the main domains and attributes shown as headings and sub-headings.

This classification provides clear structure for those main domains and attributes. Some of them are adopted and modified from the attributes of VRP taxonomy developed by Eksioglu et al. (2009). Due to the fact that this research considers one variant of VRP, that is SVRP, the number of domains and attributes considered are less in number and slightly different from in type (owing to modification) with the attributes used in the general VRP. However, they are developed after a thorough reading of each of the 49 articles identified in Sec. 3.2.

\subsection{Categorising and classifying articles}

The classification structure of SVRP is built in an arborescent way with a very close look at each article. The branching levels from top to bottom are, at most, in three levels in order to provide coherence and parsimony, yet sacrifice nothing in terms of comprehensiveness. The levelling is from abstraction to the details in order to provide clear view to the reader.

The meta-analysis was used to classify each articles based on the domains and attributes developed in Sec. 3.3. For each domain, more detailed attributes as subcategories were also derived so as to help to classify SVRP literatures. In order to present the findings, 49 articles were classified based on the domains and attributes shown in Table 3.

Table 2. Years and numbers of publication.

\begin{tabular}{lcccccccccccccccccccccccc}
\hline S/N & 1 & 2 & 3 & 4 & 5 & 6 & 7 & 8 & 9 & 10 & 12 & 13 & 14 & 15 & 16 & 17 & 18 & 19 & 20 & 21 & 22 & 23 & Total \\
\hline Year & 1963 & 1982 & 1986 & 1989 & 1990 & 1991 & 1992 & 1993 & 1995 & 2000 & 2002 & 2003 & 2004 & 2005 & 2006 & 2008 & 2009 & 2010 & 2011 & 2012 & 2013 & 2014 & \\
\hline Art.* $^{1} 1$ & 1 & 1 & 1 & 1 & 2 & 2 & 2 & 1 & 1 & 1 & 3 & 1 & 4 & 1 & 2 & 3 & 2 & 5 & 6 & 6 & 2 & 49 \\
\hline
\end{tabular}

*Art. Means the number of articles in each year. 
Table 3. Classification domains and attributes.

\begin{tabular}{lc}
\hline 1 Stochastic & 5.2 Multiple \\
1.1 Customer & 5.3 Unknown \\
1.2 Customer Demand & 6 Vehicle Capacity \\
1.3 Service Time & 6.1 Capacitated \\
1.4 Travel Time & 6.2 Uncapacitated \\
2 Solution & 6.3 Unknown \\
2.1 Exact & 7 Objective \\
2.2 Heuristic & 7.1 Minimisation \\
2.3 Others & 7.1 .1 Cost \\
Graph Representation & 7.1 .2 Distance \\
3.1 Symmetric & 7.1 .3 Time \\
3.2 Asymmetric & 7.2 Maximisation \\
3.3 Unknown & 7.3 Others \\
Data & Applied Models \\
4.1 Real & 8.1 Chance Constrained \\
& programming \\
4.2 Synthesis & 8.2 Dynamic Programming \\
4.3 No Data & 8.3 Markov chain \\
Number of Vehicle & 8.4 Stochastic with recurs \\
5.1 Single & 8.5 Others \\
\hline
\end{tabular}

\section{Results and Discussions}

Based on the meta-analysis, articles that represent rather different approaches and that address different issues related to SVRP, domains and attributes are categorised and presented in Table 4 . The authors of the 49 articles are listed in the first column of Table 4 . The remaining columns represent the domains and their corresponding attributes. While classifying, some column cells may remain vacant. This means that, the article does not address or involve that cell's attribute. But, if the article addresses the attribute in the domain corresponding to each column heading, that corresponding cell is marked with " $X$ ". The full shaded columns represent the point of branching the domains. This representation is designed due to its compactness to assign more designations in a confined space.

Based on the survey, all the articles are designated on the domain or attribute they belong to. As shown in Table 4, the articles appear to be uniformly distributed in each domain and attribute. Figure 1, shows the relative proportion of each domain and attributes. But variation is observed with each domain in the attributes. Therefore, a closer look at each domain and attribute is very important. Except the column that branches the domain, there is no column with unmarked cell. However, there are very few columns that have one, two and three marked cell(s) that confers least studied areas in the SVRP literatures.
Attribute that has only one marked cell is maximisation objective function $\left(7.2^{1}\right)$, which is obvious that most SVRPs have objective function to minimise distance, time or cost. The other attribute that has two cells marked is unknown vehicle capacity (6.3). Attribute that has only three marked cells are stochastic with recurs (8.4) in the applied mode domain.

The most important outcomes of this representation is variability of attributes in each domain. In the first domain, which considers the stochastic nature of VRP, stochastic customer demand (1.2) is the most studied attribute followed by two equally studied attributes; stochastic customer (1.1) and stochastic travel time (1.4). In the same domain, stochastic service time (1.3) is found to be the least studied. To solve SVRP models, the solution tool that mostly used is the heuristic (2.2) and exact (2.1) solution techniques. However, the use of meta-heuristics solution tools, which is represented by other solution techniques (2.3) in the study is the dominated tools used for in the solution techniques.

As explained in the literatures, most VRP models are represented in a graph. Accordingly, from those that represented their model in a graph, symmetric graph (3.1) representation is relatively used more than asymmetric graph (3.2). However, from the survey made, most SVRP literatures were studied without defining their graph representation (3.3) techniques. With regard to the nature of data used, most SVRP are studied using synthesis data (4.2) followed by no data (4.3). The synthesis data in the data attribute is dominant because most SVRP researchers either used instances from previous research or hypothetical data to test their model.

Regarding the number of vehicles considered in the SVRP articles, the dominant one is multiple vehicle (5.2) followed by single vehicle (5.1). There are also a considerable number of literatures and articles that do not specify the number of vehicles (5.3) involved in their model. However, out of 38 articles that involved either single or multiple vehicles 37 of them were studied with capacitated vehicles consideration (6.1).

With respect to objective function, the survey showed that cost minimisation (7.1.1) is the dominant objective function as compared to other attributes in the minimisation domain. Whereas maximisation objective function (7.2) was found to be the least studied attribute in the domain of objective function. However, the other types of objective function (7.3) which were studied by proofing of lemmas, propositions, and theorems are the second most dominant in this domain.

\footnotetext{
17.2 represents domain objective function and attribute maximisation labelled in Table 3 and used as column heading in Table 4 .
} 


\section{E. Berhan et al.}

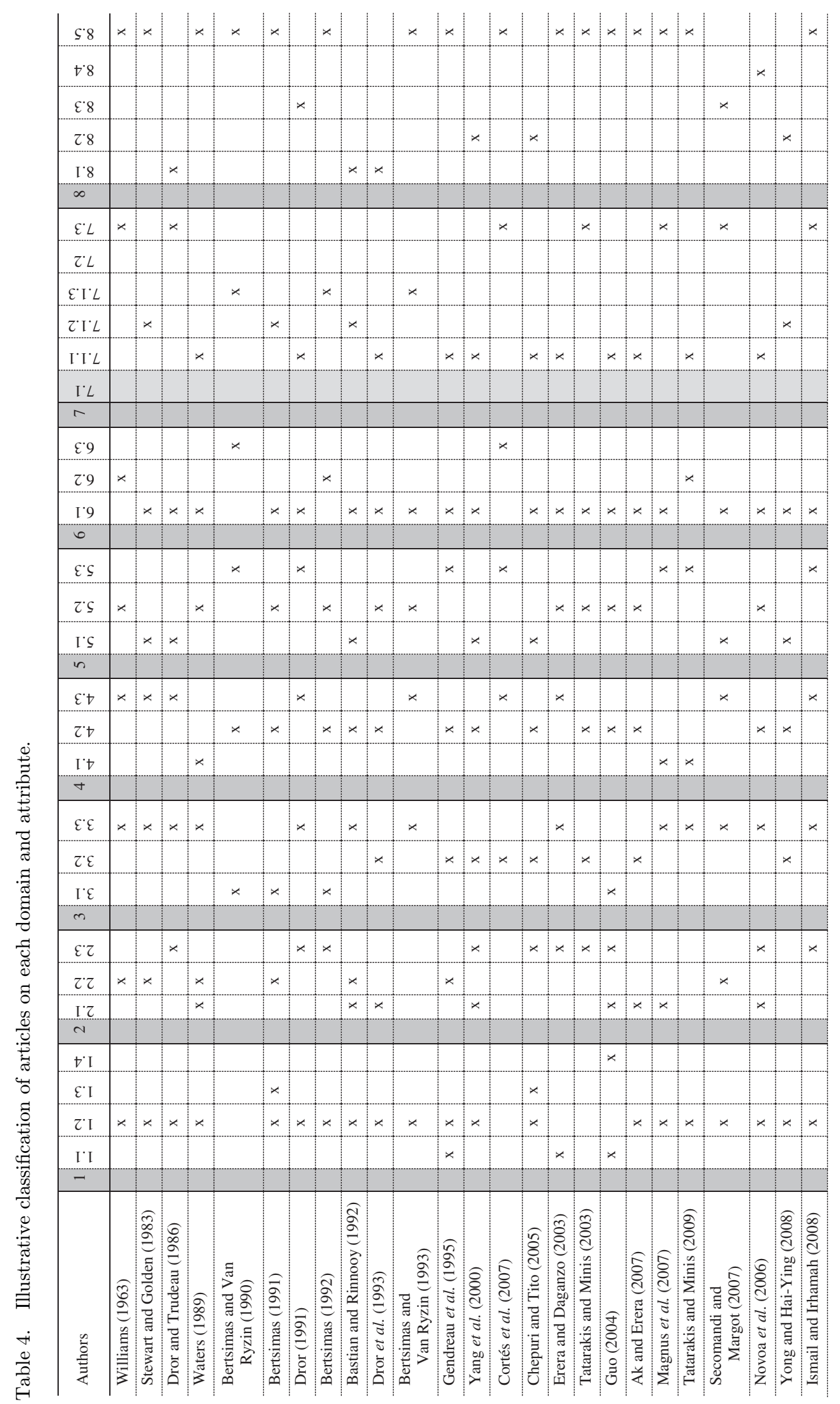




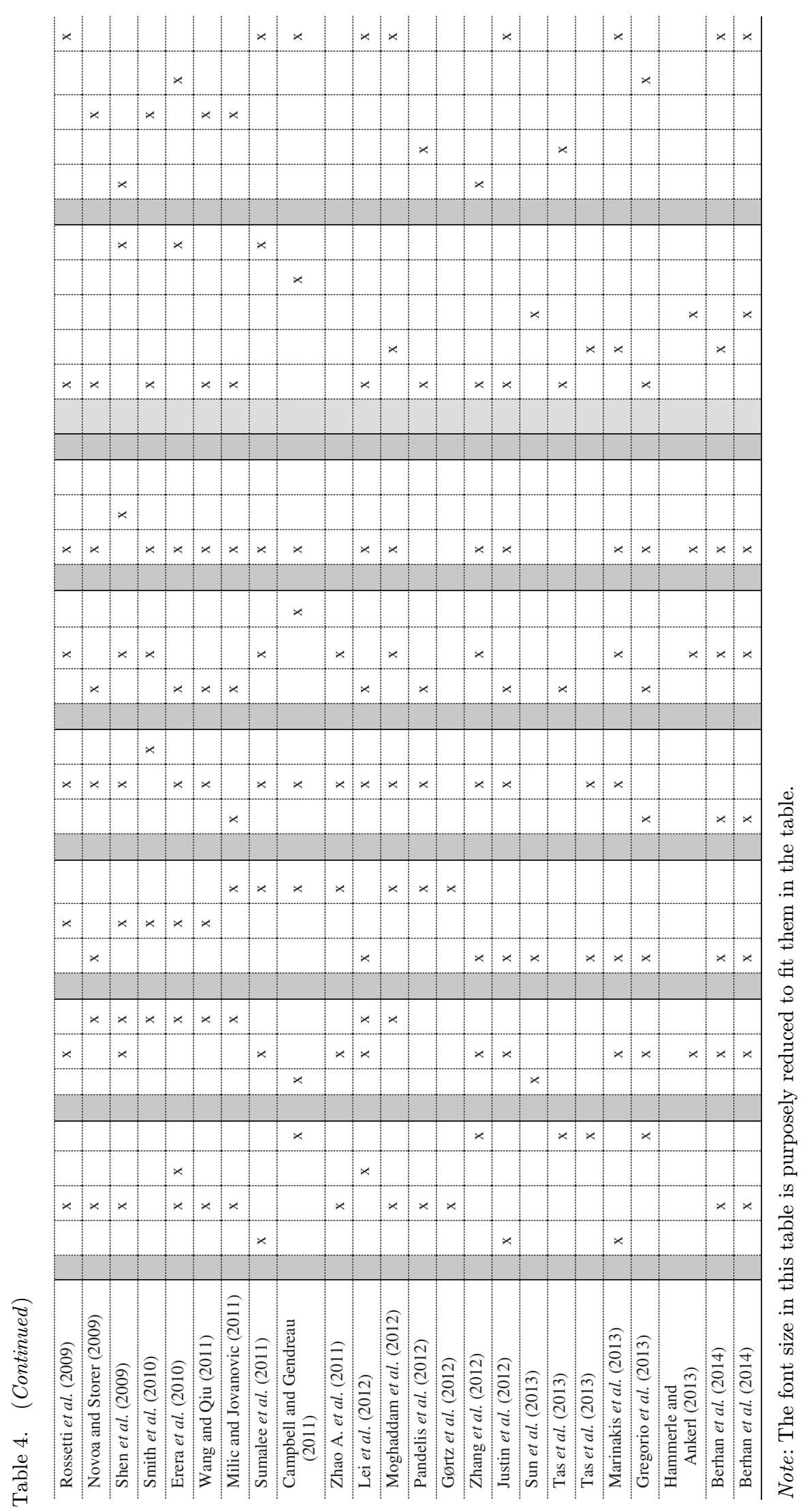




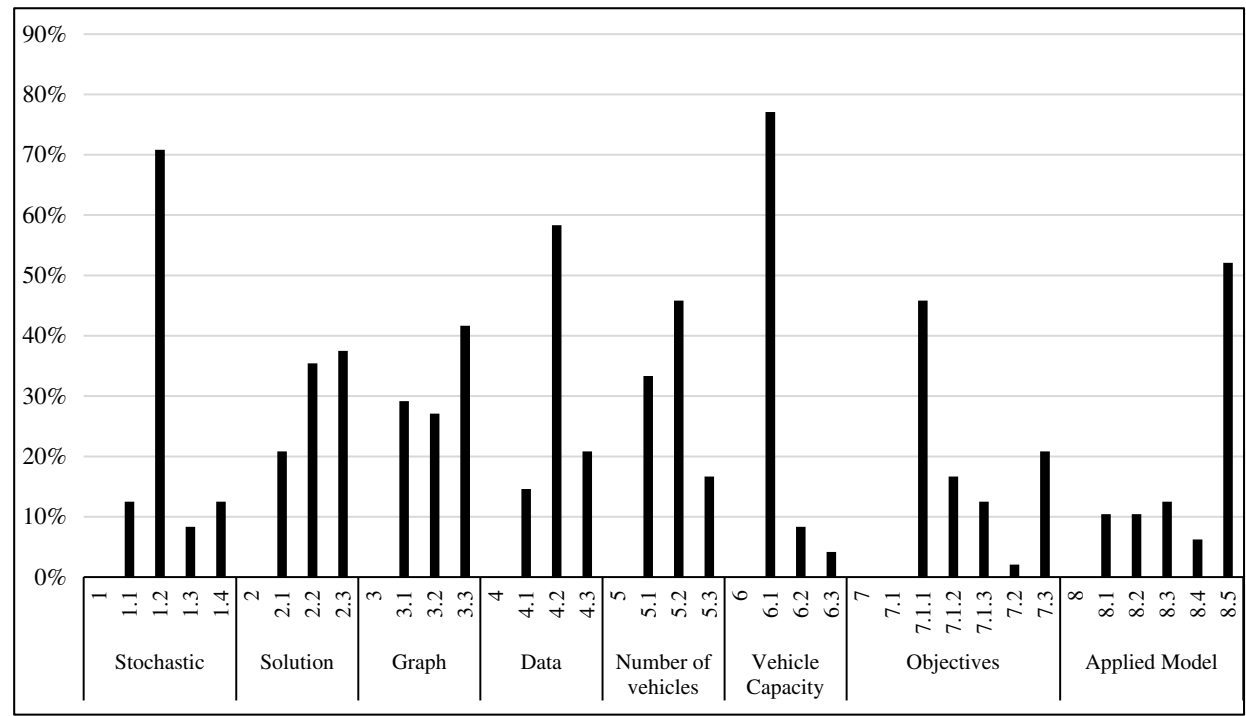

Fig. 1. The distribution of articles based on attributes.

The last domain in this paper is the type of model applied in the model formulation of SVRP studies. In this case, the majority of the studies do not identify which type of model is applied in their model formulation. It is indicated as others (8.5); and accounts 25 of the articles out of the 49 considered in the study. However, all the remaining models are nearly uniformly applied in the remaining SVRP literatures.

In SVRP studies, routing with attribute using capacitated vehicles (6.1) is very common problem formulation so as to simplify the complexity of the model. As shown in Fig. 1, the second more studied attribute is stochastic customer demand (1.2). In addition, except the unknown attributes $(2.3,3.3$, and 8.5$)$, the survey shows that the use of synthesis data (4.2), multiple number of vehicles (5.2), and objective functions with cost minimisation (7.1.1) are other attributes that were studied most in the SVRP literatures.

Attributes of stochastic service time (1.3), uncapacitated vehicle (6.2), and stochastic with recurs (8.4) are the second least studied next to maximisation objective function (7.2). Moreover, stochastic customer (1.1), stochastic travel time (1.4), the use of real data (4.1), objective function with time minimisation (7.1.3) chance constrained programming (8.1), dynamic programing (8.2), and Markov-chain applied model (8.3) were also found to be the third least studied area in SVRP literatures.

The survey result revealed that the reason for attribute no data (4.3), unknown on the graph representation (3.3), and the use of unknown applied model (8.5) is that there are many SVRP studies; that focused on proof of lemma, theorem, and proposition (Waters, 1989; Bertsimas and
Van Ryzin, 1990; Bastian and Rinnooy, 1992; Bertsimas, 1992; Dror, 1993; Dror et al., 1993).

From the survey, as presented in Table 4 and Fig. 1, the tools and techniques that are used in solution approach, graph representation, and applied models in the SVRP literatures are summarised well. Regarding, the benchmarking problems in the SVRP models, they are more versatile and diversified into various fields and cannot be organised and included in the table. However, the major benching marketing problems are briefly presented here. SVRP problems are used to model urban public bus transport system (Berhan et al., 2014), waste collection (Milijc and Jovanovijc, 2011), plant to customer distribution (Chepuri and Tito, 2005), collection and delivery of goods (Bertsimas, 1992; Erera and Daganzo, 2003), distribution of goods in the logistic problem (Rossetti et al., 2009), transport system on adverse weather condition (Sumalee et al., 2011); and one supplier and multiple retailers transportation-inventory network design (Williams, 1963; Stewart and Golden, 1983).

\section{Conclusion}

The paper tries to assess more than 3000 bibliographical entities and identified 49 articles related to SVRP. The bibliographical entities were selected from various sources published since 1963 and classified based on 8 different domains and 29 attributes. The classification has been demonstrated to be robust enough to incorporate a diverse set of SVRP literatures which were previously published as well as those expected in the future. However, there is no taxonomy that remained fixed all the 
time, it can be improved more in the future to incorporate when new insights in the area of SVRP were evolved through time. Based on the classifications, nearly all the articles are uniformly distributed across the domains and attributes, but variation is observed within each domain. From the findings, capacitated vehicle, stochastic customer demand, and synthesis data are the most dominantly studied attributes with consideration of multiple vehicles and cost minimisation objective functions. Minimisation objective function is found to be the least studied among all the attributes followed by uncapacitated vehicle and stochastic with recurs. Most SVRP studies were conducted using exact heuristic solution techniques. But the use of different meta-heuristics and other solution techniques also account for the majority of the SVRP literatures.

Moreover, from the survey made; it was realised that the stochastic consideration is not limited to customer, demand, and time; rather includes also other side constraints such as road weather conditions, workers' shift, and so on. Apart from the findings, there is no previous study that tried to classify and categorise SVRP studies except for some survey in a very few areas of focus.

Furthermore, based on the systematic review of the articles and the survey conducted, the research has also identified the following gaps in the SVRP literature which can serve as future study areas.

(1) Most SVRP studies were conducted on stochastic customer demand using objective function of cost minimisation and capacitated vehicles with synthesis data. But they were studied in a diversified way to find a solution using exact, heuristic or meta-heuristics techniques. However, SVRP which maximises vehicle utilisation, number of customer to be serviced, revenue earned, and others remain most untouched areas in the SVRP literatures.

(2) There is no SVRP literature that modelled fully the real world stochastic parameters considered in its study to solve problems using real world data. Nearly, all made an assumption to simplify and tried to fit to a probabilistic model. They tested using synthesis data or previously generated instance or using mathematical proof of lemma, theorem, and proposition.

(3) Due to the dynamic nature of side constraints in VRP or SVRP, except some open source instances developed by scholars which are modified and used in many literatures, commercial software are not readily available to fit any VRP model. This highly hinders the strategy to improve the solution methods.

\section{References}

Ak, A and AL Erera (2007). A paired-vehicle recurs strategy for the vehicle routing problem with stochastic demands. Transportation Science, 41(2), 222-237.

Balakrishnan, N (1993). Simple heuristics for the vehicle routing problem with soft time windows. Journal of Operations Research Society, 44(3), 279-287.

Baldacci, R, D Vigo and P Toth (2010). Exact solution of the capacitated vehicle routing problem, in Wiley Encyclopedia of Operations Research and Management Science, John Wiley \& Sons, Inc, US.

Bastian, C and A Rinnooy (1992). The stochastic vehicle routing problem revisited. European Journal of Operational Research, 56, 407-412.

Berhan, E, P Krömer, D Kitaw, A Abraham and V Snášel (2014). Solving stochastic vehicle routing problem with real simultaneous pickup and delivery using differential evolution. In Innovations in Bio-Inspired Computing and Applications, Advances in Intelligent Systems and Computing, Vol. 237, pp. 187-200. Switzerland: Springer International Publishing.

Bertsimas, D (1991). A vehicle routing problem with stochastic demand. Journal of Operations Research, 40(3), 554-585.

Bertsimas, D (1992). A vehicle routing problem with stochastic demand. Operations Research, 40, 574-585.

Bertsimas, D and G Van Ryzin (1990). A stochastic and dynamic vehicle routing problem in the euclidian plane. Operations Research Society of America, 39(4), 600615.

Bertsimas, D and G Van Ryzin (1993). Stochastic and dynamic vehicle routing with general demand and interarrival time distributions. Advances in Applied Probability, 25(4), 947-978.

Blanton, JL and RL Wainwright (1993). Multiple vehicle routing with time and capacity constraints using genetic algorithms, in Proceedings of the Fifth International Conference on Generic Algorithms (ICGA-93), 452-459.

Bodin, L (1975). A taxonomy structure for vehicle routing and scheduling problems. Computers and Urban Society, $1,11-29$.

Calvete, HI, G Carmen, JO Mara and SV Beln (2004). Vehicle routing problems with soft time windows: An optimization based approach. Journal of Monografas del Seminario Matemtico Garca de Galdeano, 31, 295304.

Campbell, MA and MTWB Gendreau (2011). The orienteering problem with stochastic travel and service times. Ann Oper Res, 186, 61-81.

Chenghua, S and Z Xiaofeng (2011). Research on model fitting capacity of vehicle routing problem. International Journal of Advancements in Computing Technology (IJACT), 3(11), 185-193. 
Chepuri, K and H-D-M Tito (2005). Solving the vehicle routing problem with stochastic demands using the cross-entropy method. Annals of Operations Research, 134, 153-181.

Christopher, GJ (2010). Solutions methedologies for VRP with stochastic demand, Dessertation, Iowa.

Clarke, G and J Wright (1964). Scheduling of vehicles from a central depot to a number of delivery points. Operations Research, 12, 568-581.

Cordeau, J-F, G Laporte, WPS Martin and V Daniele (2007). Vehicle Routing, in John R Birge and Vadim Linetsky, eds., Handbooks in Operations Research and Management Science, Elsevier B.V., Amsterdam, Netherlands, 366-427.

Cordeau, J-F, G Laporte and A Mercier (2001). A unifid tabu search heuristic for vehicle routing problem with time windows. Journal of Operations Research Society, 53, 928-936.

Cordeau, J-F, M Gendreau, G Laporte, J-Y Potvin and F Semet (2002). A guide to vehicle routing heuristics. Journal of the Operational Research Society, 53, $512-522$.

Cortés, C, F Ordónẽz, S Souyris and A Weintraub (2007). Routing technicians under stochastic ser vice times: A robust optimization approach. On the Sixth Triennial Symposium on Transp Ortati on Analysis, Phuket Island.

Dantzig, Gilbert, B and JH Ramser (1959), The truck dispatching problem. Management Science, 6(1), 80-91.

Desrochers, M, JK Lenstra and MWP Savelsbergh (1990). A classification scheme for vehicle routing and scheduling problems. European Journal of Operational Research, 46(3), 322-332.

Dror, M (1991). Modeling vehicle routing with uncertain demands as a stochastic program: Properties of the corresponding solution. European Journal of Operational Research, 68, 432-441.

Dror, M. (1993). Modeling vehicle routing with uncertain demands as a stochastic program: Properties of the corresponding solution. European Journal of Operational Research, 64, 432-441.

Dror, M and P Trudeau (1986). Stochastic vehicle routing with modified savings algorithm. Journal of Operational Research, 23, 228-235.

Dror, M. and P Trudeau (1989). Savings by split delivery routing. Transportation Science, 23, 141-145.

Dror, M, G Laporte and VL Francois (1993). Vehicle routing with stochastic demands and restricted failures. Methods and Models of Operations Research, 33, 271-283.

Dror, M, G Laporte and P Trudeau (1994). Vehicle routing with split deliveries. Discrete Applied Mathematics, 50, 229-254.

Dror, M and P Trudeau (1989). Savings by split delivery routing. Transportation Science, 23, 141-145.
Eksioglu, B, AV Vural and A Reisman (2009). The vehicle routing problem: A taxonomic review. Computers and Industrial Engineering, 57(4), 1472-1483.

Erera, AL and CF Daganzo (2003). A dynamic scheme for stochastic vehicle routing. Report, Georgia Institute of Technology.

Erera, AL, CJ Morales and M Savelsbergh (2010). The vehicle routing problem with stochastic demand and duration constraints. Report, The Supply Chain and Logistics Institute, Georgia Institute of Technology School of Industrial and Systems Engineering Atlanta, GA 30332-0205.

Gendreau, M, G Laporte and R Seguin (1995). An exact algorithm for the vehicle routing problem with stochastic demands and customers. Transportation Science 29, $143-155$.

Gendreau, M, G Laporte and R Séguin (1996). Stochastic vehicle routing. European Journal of Operational Research, 88, 3-12.

Ghiani, G, G Laporte, E Manni and R Musmanno (2008). Waiting strategies for the dynamic and stochastic traveling salesman problem. International Journal of Operations Research, 5(4), 233-241.

Golden, BL, S Raghavan and EA Wasil (2008). Routing a heterogeneous fleet of vehicles, in G Bruce, L, RS and W Edward, A, eds, The Vehicle Routing Problem: Latest Advances and New Challeges, Springer, US, 3-27.

Goodson, JC, JW Ohlmann and BW Thomas (2012). Cyclic-order neighborhoods with application to the vehicle routing problem with stochastic demand. European Journal of Operational Research, 217(2), 312-323.

Gørtz, IL, V Nagarajan and R Saket (2012). Stochastic vehicle routing with recurs. ICALP 2012, Part I, LNCS 7391, pp. 411-423.

Gregorio, T, LM Hvattum, K Fagerholt and J-F Cordeau (2013). Heuristics for dynamic and stochastic routing in industrial shipping. Computers and Operations Research, 40(1), 253-263.

Guo, Z (2004). A heuristic algorithm for the stochastic vehicle routing problems with soft time windows. In Evolutionary Computation. Vol. 2 of CEC 2004, Hong Kong University, China, pp. 1449-1456.

Hammerle, A and MH Ankerl (2013). Solving a vehicle routing problem with ant colony optimisation and stochastic ranking. EUROCAST, Part I, LNCS, 8111, 259-266.

Ismail, HZ and Irhamah I (2008). Solving the vehicle routing problem with stochastic demands via hybrid genetic algorithm-tabu search. Journal of Mathematics and Statistics, 4(3), 161-167.

Kallehauge, B (2008). Formulations and exact algorithms for the vehicle routing problem with time windows. Computer Operations Research 12(7), 2307-2330. 
Kenyon, SA and PD Morton (2003). Stochastic vehicle routing with random travel times. Journal of Transportation Science, 37(1), 69-82.

Kumar, NS and R Panneerselvam (2012). A survey on the vehicle routing problem and its variants. Intelligent Information Management, 4, 66-74.

Laporte, G (1992). The vehicle routing problem: An overview of exact and approximate algorithms. European Journal of Operational Research, 59, 345-358.

Laporte, G, F Louveaux and H Mercure (1989). Models and exact solutions for a class of stochastic locationrouting problems. European Journal of Operational Research, 39, 71-78.

Laporte, G, F Louveaux and VL Hamme (2002). An integer l-shaped algorithm for the capacitated vehicle routing problem with stochastic demands. Operations Research, 50, 415-423.

Lei, H, G Laporte and B Guo (2012). A generalized variable neighborhood search heuristic for the capacitated vehicle routing problem with stochastic service times. TOP, 20, 99-118.

Li, X, P Tian and SCH Lang (2010). Vehicle routing problems with time windows and stochastic travel and service times: Models and algorithm. International Journal of Production Economics, 125, 137-145.

Madsen, OG, F Ravn and M Rygaard (1995). The vehicle routing problem with time windows part ii: Genetic search. Journal of Computing, 8, 165-172.

Magnus, HL, A Lkketangen and G Laporte (2007). A branch-and-regret heuristic for stochastic and dynamic vehicle routing problems. Wiley Periodicals, Inc. NETWORKS, 49(4), 330-340.

Marinakis, Y, GR Iordanidou and M Marinaki (2013). Particle swarm optimization for the vehicle routing problem with stochastic demands. Applied Soft Computing, 13(4), 1693-1704.

Milić, P and M Jovanović (2011). The advanced system for dynamic vehicle routing in the process of waste collection. Mechanical Engineering, 9(1), 127-136.

Mingozzi, A (2003). An exact algorithm for period and multidepot vehicle routing problems., report, Department of Mathematics, University of Bologna, Bologna, Italy.

Moghaddam, FB, RR Babak and JS Sadjadi (2012). Vehicle routing problem with uncertain demands: An advanced particle swarm algorithm. Computers and Industrial Engineering, 62, 306-317.

Novoa, C and R Storer (2009). An approximate dynamic programming approach for the vehicle routing problem with stochastic demands. European Journal of Operational Research, 196, 509-515.

Novoa, C, R Berger, J Linderoth and R Storer (2006). A set-partitioning-based model for the stochastic vehicle routing problem. Technical report, Texas State University and Lehigh University.
Pandelis, DG, EG Kyriakidis and TD Dimitrakos (2012). Single vehicle routing problems with a predefined customer sequence, compartmentalized load and stochastic demands. European Journal of Operational Research, 217(2), 324-332.

Paolo, T and V Daniele (eds.) (2002). The vehicle routing problem, SIAM Monographs on Discrete Mathematics and Applications, Society for Industrial and Applied Mathematics, Philadelphia, PA.

Parragh, S, K Doerner and R Hartl (2008). A survey on pickup and delivery problems. Journal für Betriebswirtschaft, 58(1), 21-51.

Pillac, V, M Gendreau, C Guerét and LA Medaglia (2011). A review of dynamic routing problem. Interuniversity Research Center on Enterprise Network, Logistic and Transportation.

Reimann, M (2005). Analyzing a vehicle routing problem with stochastic demand using ant colony optimization. Advanced OR and AI methods in Transportation, Poznan Technical University, Poznan, 764-769.

d kalyan, in Rossetti, MD, RR Hill, B Johansson, A Dunkin and RG Ingalls (eds.) (2009). An ant based simulation optimization for vehicle problem with stochastic demands. In Proc. 2009 Winter Simulation Conf.

Secomandi, N and F Margot (2007). Reoptimization approaches for the vehicle routing problem with stochastic demands. Journals of Operations Research, $57(1), 214-230$.

Shen, Z, F Ordóñez and MM Dessouky (2009). The stochastic vehicle routing problem for minimum unmet demand. Optimization and Logistics Challenges in the Enterprise, 30, 349-371.

Smith, SL, M Pavone, F Bullos and E Frazzoli (2010). Dynamic vehicle routing with priority classes of stochastic demands. IAM Journal on Control and Optimization, 48(5), 3224-3245.

Stefan, B and K Natalia (2009). An overview on vehicle scheduling models. Decision Support \& Operations Research Lab, University of Paderborn, Str. 100, D33098 Paderborn, Warburger, Germany. Available at: https://wiwi.uni-paderborn.de/fileadmin/lehrstuehle/department-3/wiwi-dep-3-ls-5/Forschung/Publikationen/ bunte_kliewer_an_overview_on_vehicle_scheduling_models_ 2006.pdf.

Stewart, W and B Golden (1983). Stochastic vehicle routing: A comprehensive approach. European Journal of Operational Research, 14, 371-385.

Sumalee, A, K Uchida and HKW Lam (2011). Stochastic multi-modal transport network under demand uncertainties and adverse weather condition. Transportation Research Part C, 11, 338-350.

Sun, S, Z Duan and D Yang (2013). Optimal routing problem in dynamic stochastic networks. Procedia Social and Behavioral Sciences, 96(6), 817-827. 


\section{E. Berhan et al.}

Taş, D, N Dellaert, T van Woensel and T de Kok (2013). Vehicle routing problem with stochastic travel times including soft time windows and service costs. Computers and Operations Research, 40(1), 214-224.

Taş, D, M Gendreau, N Dellaert, T van Woensel and AG de Kok (2014). Vehicle routing with soft time windows and stochastic travel times: A column generation and branch-and-price solution approach. European Journal of Operational Research, 236(3), 789-799.

Taillard, E, Badezu, P, Gendreau, M, Guertin, F and P J (1997). A tabu-search heuristic for the vehicle routing problem with soft-time window. Journal of Transportation Science, 31(2), 170-186.

Tatarakis, A and I Minis (2009). Stochastic single vehicle routing with a predefined customer sequence and multiple depot returns. European Journal of Operational Research, 197, 557-571.

Wang, C and Y Qiu (2011). Vehicle routing problem with stochastic demands and simultaneous delivery and pickup based on the cross-entropy method. Advances in Automation and Robotics, 2 (LNEE 123), 55-60.

Waters, C. (1989). Vehicle-scheduling problems with uncertainty and omitted customers. Journal of the Operational Research Society, 40(12), 1099-1108.
Williams, A. (1963). A stochastic transportaion problem. Journal of Operations Research, 11(5), 759-770.

Wouter, J. (2008). Approximate models and solution approaches for the vehicle routing problem with multiple use of vehicles and time windows. Master thesis, Middle East Technical University.

Yang, W-H, M Kamlesh and BH Ronald (2000). Stochastic vehicle routing problem with restocking. Transportation Science, 34, 99-112.

Yong, P and Z Hai-Ying (2008). Research on vehicle routing problem with stochastic demand and pso-dp algorithm with inver-over operator. SETP, 28(10), 76-81.

Zhang, T, WA Chaovalitwongse and Y Zhang (2012). Scatter search for the stochastic travel-time vehicle routing problem with simultaneous pick-ups and deliveries. Computers and Operations Research, 39(10), $2277-2290$.

Zhao, Y, C Li, J-L Zhang, X Ren and W Ren (2011). Research on vehicle routing problem with stochastic demand based on multi-objective method. ICIC 2011, LNCS 6838, 153-161, 2011. 